
and Its Applications

18-30 November 2019

Chaired by Prof. Geert Verdoolaege

\title{
Information Theory in Computational Biology
}

\author{
Pritam Chanda, PhD \\ Research Scientist, Data Science \& Informatics, \\ Corteva AgriScience R\&D, Indianapolis, IN, USA
}




\section{Talk Organization}

- Information theoretic measures : Definitions and terminology

- Gene regulatory network inference

- Identifying disease associated genetic variations

- Biological Sequence Analysis: alignment free phylogeny 


\section{Information Theory}

- "A Mathematical Theory of Communication" - Claude Shannon (1948).

- Data transmission through (noisy) channels

- Diverse applications : physics, computer science, statistics, economics, neurobiology, genetics, epidemiology, ecology, bioinformatics and computational biology.

- Information theory and the living system - Lila L Gatlin, 1972

- Information content of DNA. (J. Theor. Biol. 1966)

- Information content of DNA. II. (J. Theor. Biol. 1968) 


\section{Information theoretic measures}

- Entropy

$$
H(f)=-E[\log f(x) \hat{]}
$$

Let $X$ be a random variable which takes its values from $\chi$ and its probability mass function be:

$$
p(x)=\operatorname{Pr}\{X=x\}, \quad x \in \chi
$$

$H(X)=-\sum_{x} p(x) \log p(x)=E[-\log p(x)]$

- Joint Entropy

$$
H(X, Y)=-\sum_{x \in X} \sum_{y \in Y} p(x, y) \cdot \log p(x, y)
$$

- Relative Entropy (KLD)

$$
D(p \| q)=\sum_{x \in \chi} p(x) \cdot \log \frac{p(x)}{q(x)}
$$




\section{Information theoretic measures}

- Mutual Information (MI) $\quad I(X ; Y)=\sum_{X \in X} \sum_{y \in Y} p(x, y) \cdot \log \frac{p(x, y)}{p(x) \cdot p(y)}$

$$
=D(p(x, y) \| p(x) \cdot p(y))
$$

$$
I(X ; Y)=H(X)+H(Y)-H(X, Y)
$$

- Conditional Mutual Information (CMI)

$$
I(X, Y \mid Z)=H(X \mid Z)+H(Y \mid Z)-H(X, Y \mid Z)
$$

$I(X ; Y \mid Z)-I(X ; Y)$, is called Interaction information 


\section{Information theoretic measures}

- K-Way interaction Information (KWII)

- Amount of information (synergy or redundancy) that is present in the set of variables, which is not present in any subset of these variables

$$
\begin{aligned}
K W I I\left(X_{1} ; X_{2} ; X_{3}\right)= & -H\left(X_{1}\right)-H\left(X_{2}\right)-H\left(X_{3}\right) \\
& +H\left(X_{1}, X_{2}\right)+H\left(X_{1}, X_{3}\right)+H\left(X_{2}, X_{3}\right) \\
& -H\left(X_{1}, X_{2}, X_{3}\right)
\end{aligned}
$$

For the $n$-variable case on the set $v=\left\{X_{1} X_{2}, \ldots, X_{n}\right\}$

$$
\mathrm{KWII}(v) \equiv-\sum_{T \subseteq v}(-1)^{\left|{ }^{|v|}\right| T \mid} H(T)
$$




\section{Information theoretic measures}

Total Correlation Information: Total amount of information shared among the variables in the set.

$\operatorname{TCI}\left(X_{1}, X_{2}, \ldots, X_{n}\right)=\left[\sum_{l=1}^{n} H\left(X_{t}\right)\right]-H\left(X_{1}, X_{2}, \ldots, X_{n}\right)$

$T C I\left(X_{1}, X_{2}, \ldots, X_{n}\right)=\sum_{\nu \in\left\{X_{1}, X_{2}, \ldots, x_{n}\right\},|\nu| \geq 2} K W I I(\nu)$

Phenotype Association Information: Total amount of information shared among the variables in the set with respect to a class variable.

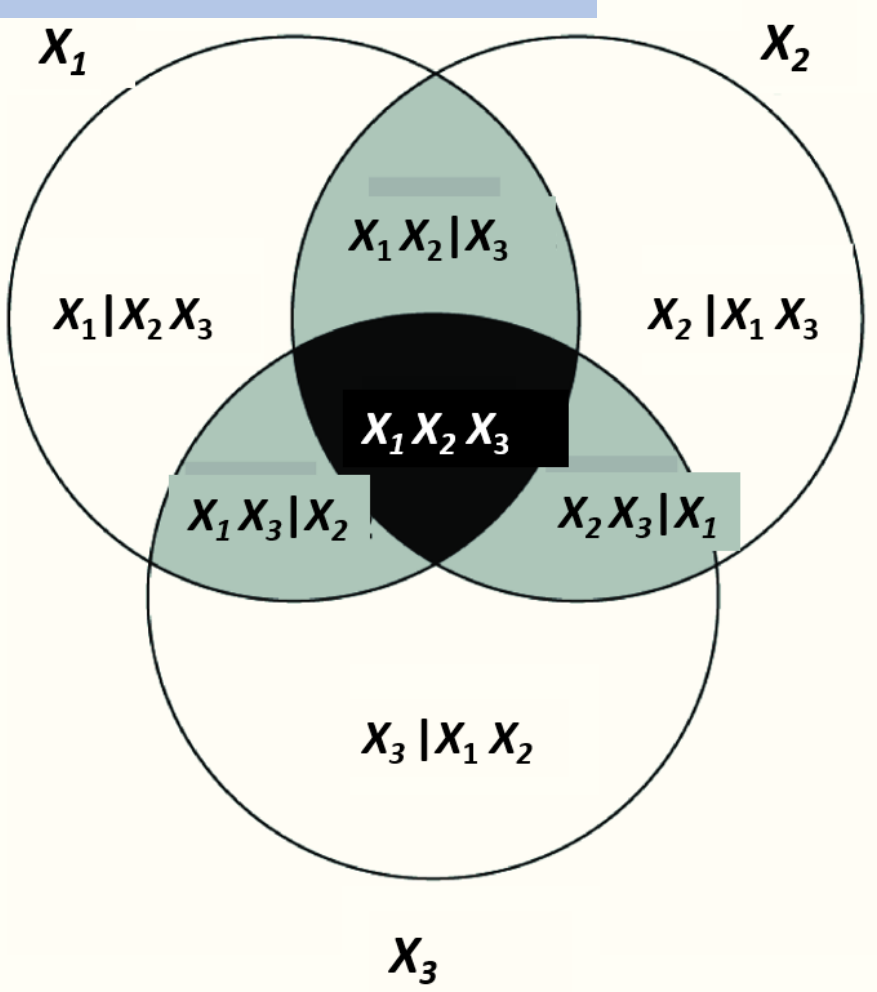

Bell AJ at. al. Proc. 4th Int. Symp. Independent Component Analysis and Blind Source Separation, 2003

Chanda et al. Am J Hum Genet. 2007, 81 (5): 939-963

$$
\operatorname{PAI}\left(X_{1}, X_{2}, \ldots, X_{K}, P\right)=\operatorname{TCI}\left(X_{1}, X_{2}, \ldots, X_{K}, P\right)-\operatorname{TCI}\left(X_{1}, X_{2}, \ldots, X_{K}\right)
$$




\section{Gene Regulatory Network Inference}

Samples

- Reverse engineering transcriptional regulatory network from expression data

- Genome-wide clustering of gene expression profiles

- Coarse representation of genes that are co-regulated together

- Gene-gene interaction network/graph from expression data

- A graph $G(V, E)$ represents a network where $V$ denotes a set of genes and $E$ denotes a set of regulatory relationships between genes.

- Each gene/transcription factor is a node in the network/graph

- Each edge models the statistical dependency between the two nodes.

- If gene $x$ shares a regulator relationship with gene $y$, then there exists an edge between $x$ and $y$, i.e $(x---y)$.

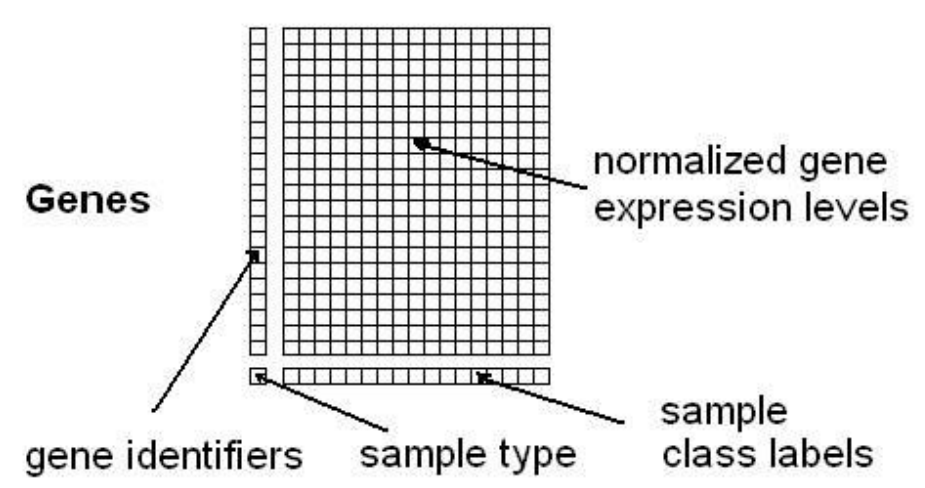

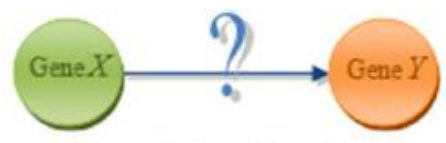

"Is there a regulatory interaction from gene $X$ to gene $Y$
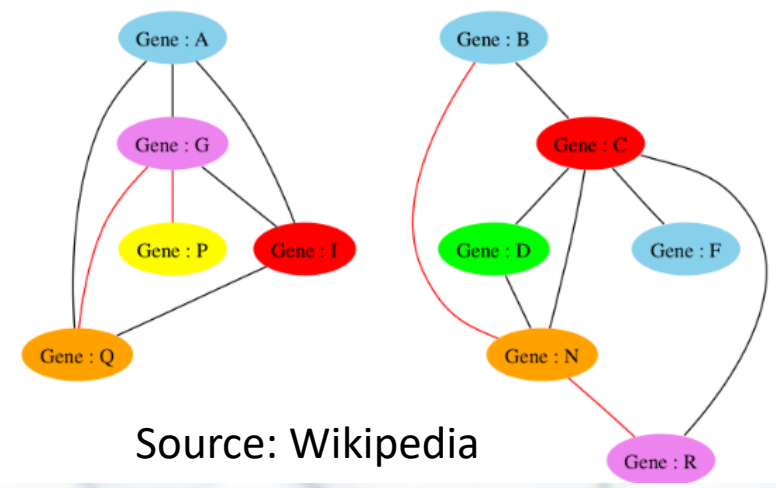

Source: Wikipedia

Gene : R

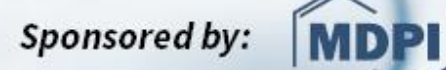




\section{Mutual Information (MI) between genes}

$I\left(G_{i}, G_{j}\right)=\sum_{g_{i} \in \Omega} \sum_{g_{i} \in \Omega} p\left(g_{i}, g_{j}\right) \log \left(\frac{p\left(g_{i}, g_{j}\right)}{p\left(g_{i}\right) p\left(g_{j}\right)}\right)$

between the gene random variables $G_{i}$ and $G_{j}$

Pairwise measurements for every pair of genes in the expression matrix

Mutual information is zero if $G_{i}$ and $G_{j} \quad$ are independent

Relevance Networks : if the mutual information between the expression levels of two genes is higher than a threshold, it is more likely that they have a biological relationship

$$
\text { edge iff : } I\left(G_{i} ; G_{j}\right) \geq \text { threshold }
$$




\section{CLR (context likelihood of relatedness)}

Considers the background distribution of the MI values
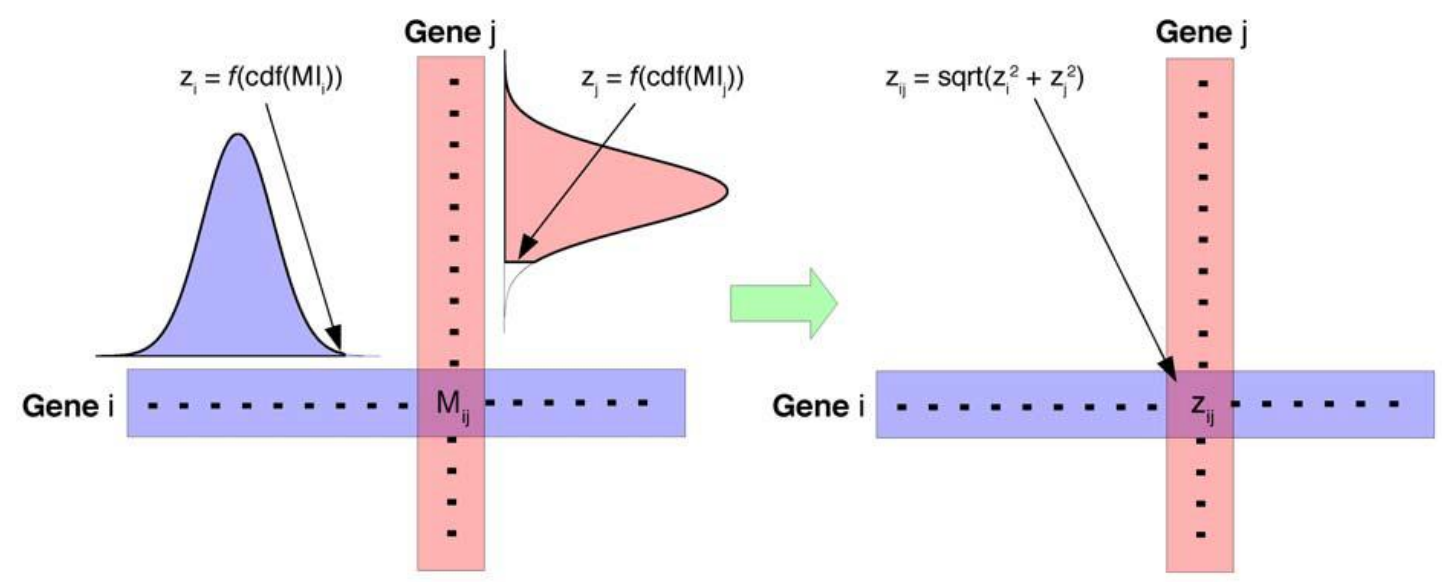

$z_{i}=\max _{j}\left(0, \frac{I\left(G_{i} ; G_{j}\right)-\mu_{i}}{\sigma_{i}}\right)$

Mean and standard deviation of MI values $\left\{I\left(G_{i} ; G_{k}\right)\right\}$

$$
\text { score }_{i, j}=\sqrt{z_{i}^{2}+z_{j}^{2}}
$$

Compares the MI between a pair of genes $G_{i}$ and $G_{j}$ to the background distribution of mutual information scores for all possible gene pairs that include either $G_{i}$ or $G_{j}$.

At a $60 \%$ true positive rate, CLR identified 1,079 regulatory interactions (741 novel predictions) in E coli. 


\section{ARACNE (Algorithm for the Reconstruction of Accurate Cellular Networks)

Compute pairwise Mutual Information for every pair of genes

- Estimated as

$$
\hat{I}\left(G_{i} ; G_{j}\right)=\frac{1}{M} \sum_{i} \log \left(\frac{f\left(G_{i}, G_{j}\right)}{f\left(G_{i}\right) f\left(G_{j}\right)}\right)
$$

- $f($.$) estimated using Gaussian Kernel Density estimation$

Filter Interactions

- $I\left(G_{i} ; G_{j}\right)>I_{t h}$ are only retained.

- $I_{t h}$ : shuffling the gene expressions to get null distribution of mutual information

Data Processing Inequality: $I\left(G_{i} ; G_{j}\right) \leq \min \left(I\left(G_{i} ; G_{k}\right), I\left(G_{k} ; G_{j}\right)\right)$

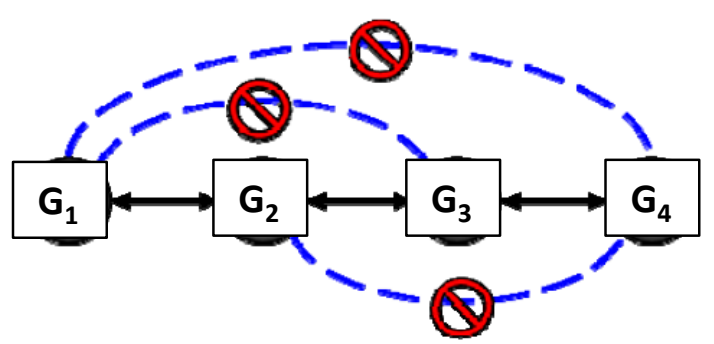

$G_{1}, G_{2}, G_{3}$, and $G_{4}$ are connected in a linear chain relationship. $\mathrm{I}\left(G_{1} ; G_{2}\right)>\mathrm{I}\left(G_{1} ; G_{3}\right)$ and $\mathrm{I}\left(G_{2}, G_{3}\right)>\mathrm{I}\left(G_{1}, G_{3}\right)$ : remove $G_{1}---G_{3}$ $I\left(G_{2}, G_{3}\right)>I\left(G_{2}, G_{4}\right)$ and $I\left(G_{3}, G_{4}\right)>I\left(G_{2}, G_{4}\right)$ : remove $G_{2}--G_{4}$

$I\left(G_{1}, G_{2}\right)>I\left(G_{1}, G_{4}\right)$ and $I\left(G_{2}, G_{4}\right)>I\left(G_{1}, G_{4}\right)$, : remove $G_{1}^{---} G_{4}$ $I\left(G_{1}, G_{3}\right)>I\left(G_{1}, G_{4}\right)$ and $I\left(G_{3}, G_{4}\right)>I\left(G_{1}, G_{4}\right)$. 


\section{Other notable methods}

- MRNET : based on maximum relevance/minimum redundancy (MRMR) principle [Meyer PE, et al. EURASIP J. Bioinf. Syst. Biol 2007.]

- Predictive Minimum Description Length (PMDL)

- Used minimum description length (MDL) to find a threshold for $\mathrm{MI}$, then $\mathrm{CMI}$ to infer regulatory relationships [Chaitankar V, et al. BMC Syst. Biol 2010;4(Suppl 1):S7.]

- PCA-CMI : higher order $\mathrm{CMI}$ and path consistency algorithm to prune a MI based gene network [zhang X, et al. Bioinformatics 2012;28(1):98104.] 


\section{Identifying Disease Associated Statistical Interactions}

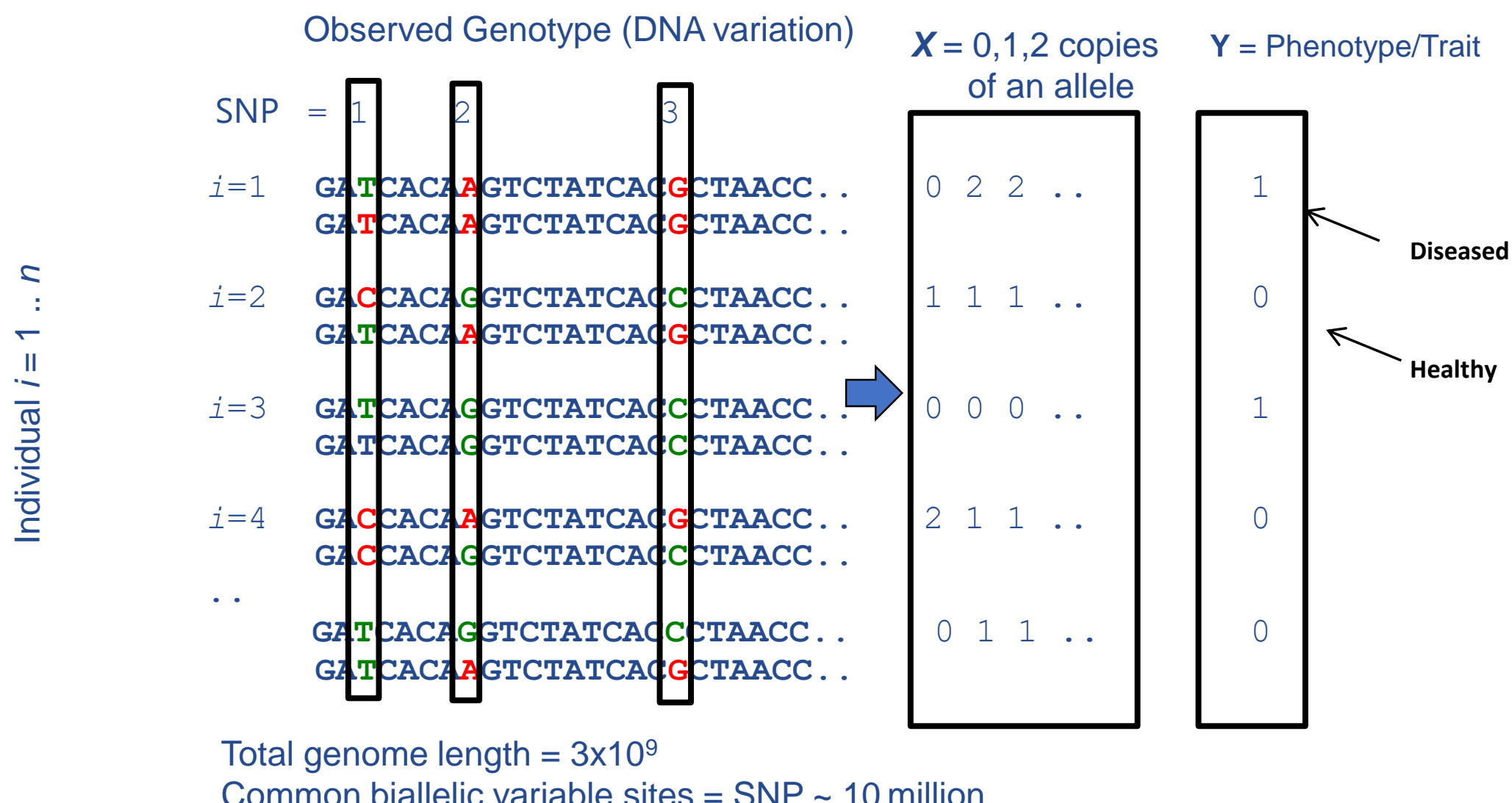




\section{Gene-Gene Interactions (GGI) and Gene- Environment Interactions(GEI)}

- Analyzed and visualized using KWII and TCl. [Chanda et al. Am J Hum Genet. 2007, 81 (5): 939-963]

- AMBIENCE: greedy algorithm to identify GEI and GGI in genome-wide data using KWII and PAI [ Chanda et. Al (2008) Genetics. 2008, 180 (2)].

- Higher power in identifying interactions [Sucheston et. al. BMC Genomics , 2010, vol. 3 pg. 487]

- CHORUS: Higher order interaction identification algorithm for quatitative traits [P Chanda, BMC genomics $10(1), 509$ ]

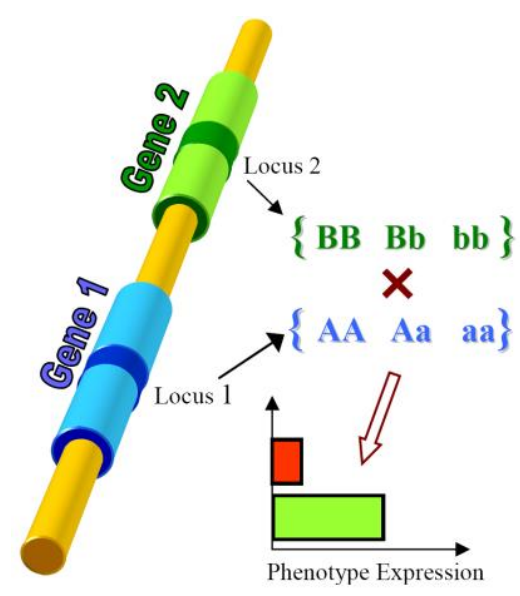




\section{Epistasis networks}

- Graph $G=\{V, E\} . V=\{S N P s\} . E=\{$ Edges between SNPs $\}$.

- Initial weight for each SNP using $\mathrm{MI}: I\left(S N P_{i}, P\right), \mathrm{P}=$ Bladder cancer susceptibility. [Hu T, 2011 BMC Bioinformatics 12:364].

- Edge between $S N P_{i}$ and $S N P_{j}: K W I I\left(S N P_{i} ; S N P_{j} ; P\right) \geq$ threshold

- Permutations to assess threshold and significance.

- Network Properties: connected components size, vertex degree distributions.

- Approximately scale free.

- Multi-SNP disease associations (connected components of size $>=2$ )

- existence of main effects does not

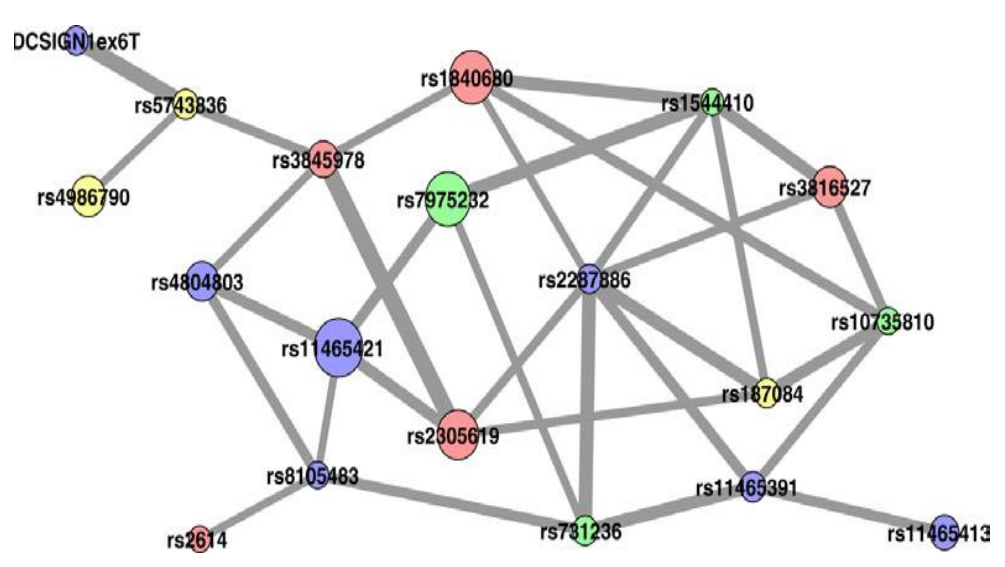
necessarily correlate with the occurrence of interactions. 


\section{Alignment free genome comparison}

- Why alignment free ?

- Assumption that similar sequences will have conserved sequence stretches is often violated.

- Accuracy of sequence alignment methods drops off rapidly when the sequence identity falls below a certain critical point.

- Computationally intensive and memory constrained for use with

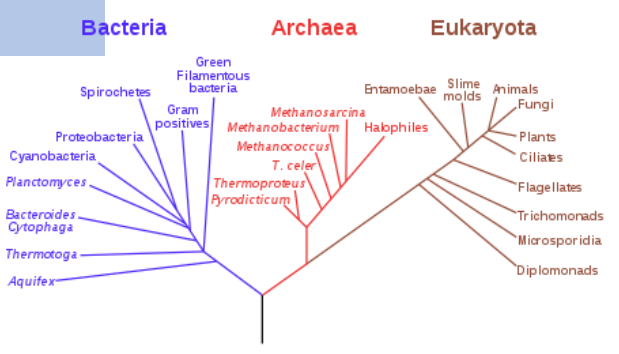

A phylogenetic tree Source: Wikipedia multi-genome-scale sequence data.

- Alignment methods often have arbitrary parameters (gap penalty, various substitution matrices etc.).

- Often difficult to align genomes, each species can have its own gene content.

- Alignment-free approaches to sequence comparison :

method of quantifying sequence similarity that does not use or produce alignment (assignment of residue-residue correspondence) at any step of algorithm application 


\section{Alignment free genome comparison using feature frequency profiles

- Determining the similarity/dissimilarity between a pair of genomes

- "Words" from genome - sliding window of length $l$ from position 1 to $n-l+1$

A GGGTAAAACTGTGTGCCAAATGC Sliding windows of length 9

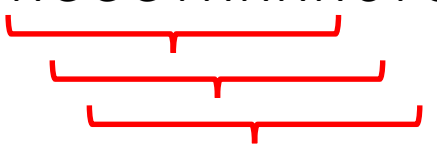
words $=\{$ AGGGTAAAA, GGGTAAAAC,$\ldots$, GCCAAATGC $\}$

- Count the frequency of each "word" (l-mer)

- Feature Frequency Profile (FFP) for a genomic sequence

- Distance between two genomic FFPs $\mathrm{P}_{l}$ and $\mathrm{Q}_{l}$ :

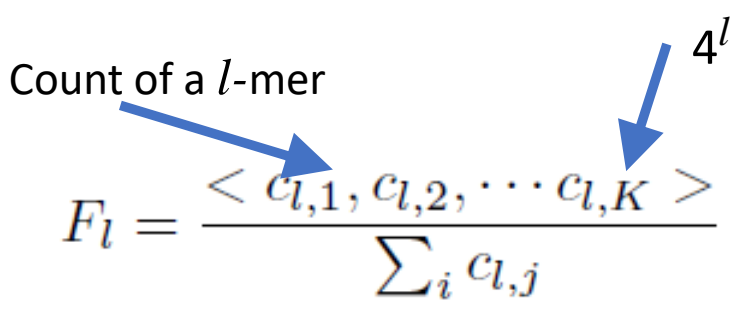

Jensen Shannon Divergence (JSD)

$$
J S D\left(P_{l}, Q_{l}\right)=\frac{K L\left(P_{l}, M_{l}\right)+K L\left(Q_{l}, M_{l}\right)}{2} \quad M_{l}=\frac{P_{l}+Q_{l}}{2}
$$

Symmetric

$$
K L\left(P_{l}, M_{l}\right)=\sum_{i=1}^{K} p_{i j} \log \frac{p_{i j}}{m_{l i}}
$$

Kullback Leibler Divergence (KLD) Asymmetric 


\section{Alignment free genome comparison using feature frequency profiles}
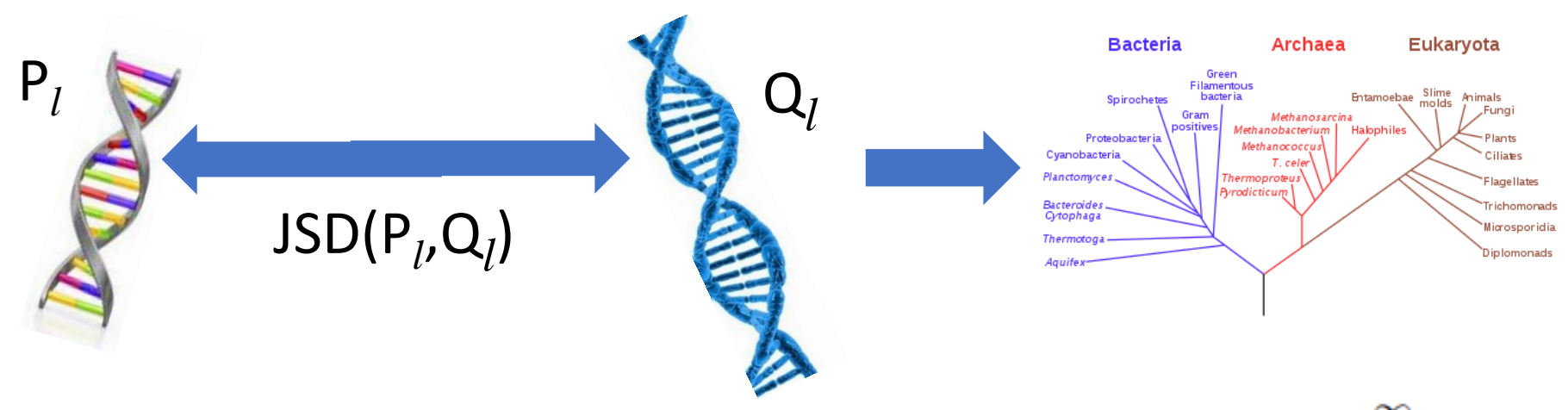

How to find optimal $l$ ?

$C R E(l)=\sum_{i=1}^{\infty}\left|K L\left(\hat{F}_{i}, F_{i}\right)\right|$
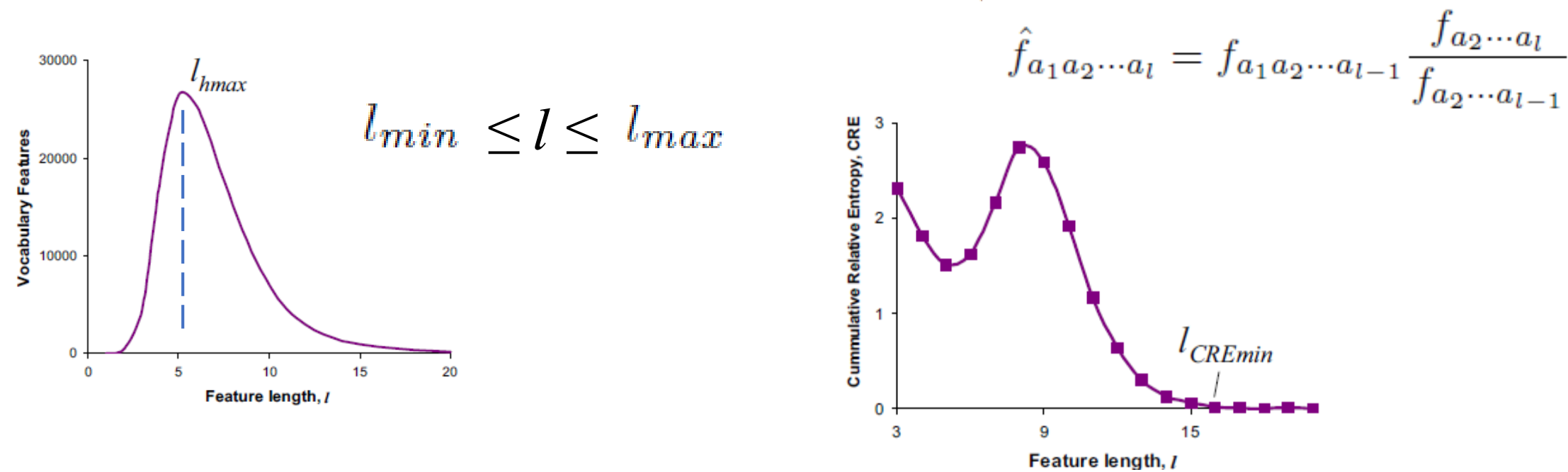
on Entropy and Its Applications

18-30 November 2019; Chaired by Prof. Geert Verdoolaege 


\section{Thank You!}

